\title{
New Optimizations and Heuristics for Determinization of Büchi Automata *
}

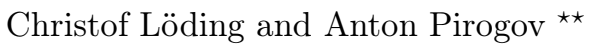 \\ RWTH Aachen University, Templergraben 55, 52062 Aachen, Germany \\ \{loeding, pirogov\}@cs.rwth-aachen. de
}

\begin{abstract}
In this work, we present multiple new optimizations and heuristics for the determinization of Büchi automata that exploit a number of semantic and structural properties, most of which may be applied together with any determinization procedure. We built a prototype implementation where all the presented heuristics can be freely combined and evaluated them, comparing our implementation with the state-ofthe-art tool spot on multiple data sets with different characteristics. Our results show that the proposed optimizations and heuristics can in some cases significantly decrease the size of the resulting deterministic automaton.
\end{abstract}

Keywords: Büchi $\cdot$ parity $\cdot$ automata $\cdot$ determinization $\cdot$ heuristics

\section{Introduction}

Nondeterministic Büchi automata (NBA) [4] are a well-established formalism for the representation of properties of non-terminating executions of finite state programs, and arise often as a low-level representation obtained by translation of some formula describing the desired behaviour in a logic like e.g. LTL [33]. In the field of model checking, Büchi automata are an important tool in verification algorithms for finite state systems (see, e.g., [2]). In order to capture the full class of regular $\omega$-languages, Büchi automata need to be nondeterministic. In some applications, however, the algorithms require the property to be represented by a deterministic automaton. For example, in probabilistic model checking, the natural product of an automaton with a Markov chain requires the automaton to be deterministic in order to produce again a Markov chain. Therefore, many algorithms in this setting need deterministic automata as input (see, e.g., [2, Section 10.3]). Another example is the problem of synthesis of finite state systems from $\omega$-regular specifications, where the specification can be translated into a game over a finite graph based on a deterministic parity automaton $[40,27]$.

\footnotetext{
* The final authenticated publication is available online at https://doi.org/10.1007/978-3-030-31784-3_18

** This work is supported by the German research council (DFG) Research Training Group 2236 UnRAVeL
} 
For this reason, the determinization of Büchi automata is a well-researched problem. In order to obtain a deterministic automaton model that is as expressive as nondeterministic Büchi automata, one needs to use a more expressive acceptance condition such as Muller, Rabin, Streett, or parity conditions (see, e.g., $[38,41])$. The first determinization construction by McNaughton [26] resulted in doubly-exponential Muller automata, whereas the first asymptotically optimal construction was presented by Safra [35], which yields Rabin automata with $2^{\mathcal{O}(n \log n)}$ states. Since then, modifications of Safra's construction have been proposed in order to improve the constants in the exponent of the state complexity [32,36], resulting in a construction for which tight lower bounds exist $[7,36]$. In particular, Piterman was the first to present a direct construction of a deterministic parity automaton (DPA) [32], which is described in a slightly different way in [36]. Another variant of Safra's construction is presented in [34].

While the Safra construction (and its variants) is the most famous determinization construction for Büchi automata, there is another approach which can be derived from a translation of alternating tree automata into nondeterministic ones by Muller and Schupp [31]. Determinization constructions for Büchi automata based on the ideas of Muller and Schupp have been described in $[19,14,13]$. The two types of constructions, Safra and Muller/Schupp, are unified in $[25]$.

While in theory, constructions with tight upper and lower bounds have been achieved, there is a lot of room for optimizations when implementing determinization constructions. A first implementation of Safra's construction in the version of [32] is ltl2dstar, presented in [20]. While ltl2dstar already implements some optimizations and heuristics in order to reduce the size of the output automaton, the resulting automata are still quite large, even for small input Büchi automata. The current state-of-the-art tool for determinization of Büchi automata is part of the library spot [9], which implements the variant of Safra's construction presented in [34]. Furthermore, spot also implements more optimizations to reduce the size of the output automata.

The contribution of this paper is the identification of new heuristics for reducing the size of DPA produced by determinization from Büchi automata and a simple framework for their implementation. More specifically, our contributions can roughly be described as follows:

- We present a modularized version of the construction in [25] which enables the integration of SCC-based heuristics.

- By exploiting properties of the used construction, we make stronger use of language inclusions between states of the given NBA (e.g. obtained by simulation), permitting to use inclusions between states in the same SCC.

- We treat specific types of SCCs in the NBA in a special way, namely those without accepting states, those with only accepting states, and those with a deterministic transition relation.

- The construction from [25] leaves some freedom in the choice of successor states. Our implementation admits different options for this succes- 
sor choice, in particular those leading to the constructions of Safra and of Muller/Schupp.

Furthermore, we have an optimization that exploits this freedom of successor selection by checking whether admissible successor states have already been constructed before adding a new state.

- We make use of language equivalences of states in the constructed DPA in order to remove some SCCs of the resulting DPA. These language equivalences are derived during construction time from the powerset automaton for the given NBA.

- We propose to use known minimization techniques as post processing, which to the best of our knowledge, have not yet been used in this context. We first minimize the number of priorities of the DPA using an algorithm from [5], and then reduce the size of the resulting DPA by Hopcroft's algorithm [17], treating it as a finite automaton that outputs the priorities.

- We have evaluated the combination of different heuristics on different types of data-sets (randomly generated NBAs, NBAs constructed from random LTL formulas, NBAs generated from some families of LTL formulas taken from the literature, and NBAs obtained from specifications of the competition SYNTCOMP [18]), and compared the size of the resulting automata with the ones produced by spot.

This work is organized as follows. After some preliminaries in Section 2, we sketch the general construction on which we based our implementation in Section 3, and then describe our optimizations and heuristics in Section 4. In Section 5 we present our experiments and in Section 6 we conclude.

\section{Preliminaries}

First we briefly review basic definitions concerning $\omega$-automata and $\omega$-languages. If $\Sigma$ is a finite alphabet, then $\Sigma^{\omega}$ is the set of all infinite words $w=w_{0} w_{1} \ldots$ with $w_{i} \in \Sigma$. For $w \in \Sigma^{\omega}$ we denote by $w(i)$ the $i$-th symbol $w_{i}$. For convenience, we write $[n]$ for the set of natural numbers $\{1, \ldots, n\}$. A Büchi automaton $\mathcal{A}$ is a tuple $\left(Q, \Sigma, \Delta, Q_{0}, F\right)$, where $Q$ is a finite set of states, $\Sigma$ a finite alphabet, $\Delta \subseteq Q \times \Sigma \times Q$ is the transition relation and $Q_{0}, F \subseteq Q$ are the sets of initial and final states, respectively. When $Q$ is understood and $X \subseteq Q$, then $\bar{X}:=Q \backslash X$. We write $\Delta(p, a):=\{q \in Q \mid(p, a, q) \in \Delta\}$ to denote the set of successors of $p \in Q$ on symbol $a \in \Sigma$, and $\Delta(P, a)$ for $\bigcup_{p \in P} \Delta(p, a)$. A run of an automaton on a word $w \in \Sigma^{\omega}$ is an infinite sequence of states $q_{0}, q_{1}, \ldots$ starting in some $q_{0} \in Q_{0}$ such that $\left(q_{i}, w(i), q_{i+1}\right) \in \Delta$ for all $i \geq 0$. As usual, an automaton is deterministic if $\left|Q_{0}\right|=1$ and $|\Delta(p, a)| \leq 1$ for all $p \in Q, a \in \Sigma$, and nondeterministic otherwise. In this work, we assume Büchi automata to be nondeterministic and refer to them as NBA. A (transition-based) deterministic parity automaton (DPA) is a deterministic automaton $\left(Q, \Sigma, \Delta, Q_{0}, c\right)$ where instead of $F \subseteq Q$ there is a priority function $c: \Delta \rightarrow \mathbb{N}$ assigning a natural number to each transition.

A run of an NBA is accepting if it contains infinitely many accepting states. A run of a DPA is accepting if the smallest priority of transitions along the run 
which appears infinitely often is even. An automaton $\mathcal{A}$ accepts $w \in \Sigma^{\omega}$ if there exists an accepting run on $w$, and the language $L(\mathcal{A}) \subseteq \Sigma^{\omega}$ recognized by $\mathcal{A}$ is the set of all accepted words. If $P$ is a set of states of an automaton, we write $L(P)$ for the language accepted by this automaton with initial state set $P$. For sets consisting of one state $q$, we write $L(q)$ instead of $L(\{q\})$. We sometimes refer to states of a DPA that is obtained by a determinization construction as macrostates to distinguish them from the states of the underlying Büchi automaton.

We write $p \stackrel{x}{\rightarrow} q$ if there exists a path from $p$ to $q$ labelled by $x \in \Sigma^{+}$and $p \rightarrow q$ if there exists some $x$ such that $p \stackrel{x}{\rightarrow} q$. The strongly connected component $(S C C)$ of $p \in Q$ is $\operatorname{scc}(p):=\{q \in Q \mid p=q$ or $p \rightarrow q$ and $q \rightarrow p\}$. The set $\operatorname{SCCs}(\mathcal{A}):=\{\operatorname{scc}(q) \mid q \in Q\}$ is the set of all SCCs and partitions $Q$. A component $C \in \operatorname{SCCs}(\mathcal{A})$ is trivial if $C=\{q\}$ for some $q \in Q$ and $q \not \rightarrow q$. $C$ is bottom if $p \rightarrow q$ implies $q \in C$ for all $p \in C$ and $q \in Q$. In a Büchi automaton, $C$ is rejecting if it is trivial or contains no accepting states, and accepting if it is not trivial and all cycles inside $C$ contain an accepting state. If an SCC is neither accepting or rejecting, it is mixed. Notice that rejecting and accepting components are often called inherently weak in the literature (e.g. [3]). Finally, $C$ is deterministic if $|\Delta(p, a) \cap C| \leq 1$ for all $p \in C$ and $a \in \Sigma$. An NBA is limit-deterministic, if all its non-rejecting SCCs are deterministic and cannot reach non-deterministic SCCs again.

\section{Construction}

For our prototype implementation, we applied new optimizations and heuristics to an adaptation of a recent generalized determinization construction from NBA to DPA that was presented in [25]. This construction unifies the constructions of Safra and of Muller and Schupp, and also introduces new degrees of freedom, which we exploit in one of our heuristics (see Section 4.2).

Let $\mathcal{A}=\left(Q, \Sigma, \Delta, Q_{0}, F\right)$ be the NBA to be determinized. The macrostates $(\alpha, t)$ in the deterministic automaton (called ranked slices) are tuples of disjoint non-empty sets $t:=\left(S_{1}, S_{2}, \ldots, S_{n}\right)$ equipped with a bijection $\alpha:[n] \rightarrow[n]$ that assigns to each set $S_{i}$ the rank $\alpha(i)$. These ranks are used to define the priorities of the transitions. When reading symbol $a \in \Sigma$ in macrostate $(\alpha, t)$, the successor $\left(\alpha^{\prime}, t^{\prime}\right)$ is obtained by applying the successive operations step, prune, merge and normalize. An overview of the complete transition is sketched in Figure 1 and we refer to [25] for more details.

\subsection{A modular variant of the construction}

We present a generalization of the construction above which offers a clean framework to implement our heuristics, specifically the heuristics that exploit the SCC structure of the Büchi automaton. This variant of the construction runs multiple interacting instances of the construction above in parallel, where each instance is essentially a ranked slice as above, but manages only an SCC of the Büchi automaton (or a union of SCCs). A macrostate in this modular construction consists of not one, but (in general) multiple tuples with a global ranking function (see illustration in Figure 2). Furthermore, for some heuristics it is not needed 


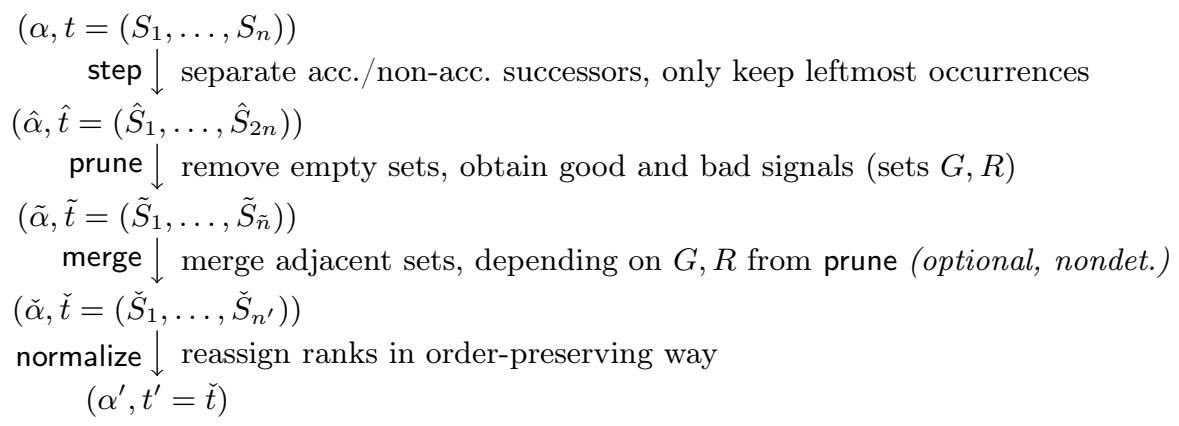

Fig. 1. Sketch of some transition $(\alpha, t) \stackrel{a \in \Sigma}{\rightarrow}\left(\alpha^{\prime}, t^{\prime}\right)$ of the determinization construction. The nondeterminism in the optional merge operation enables the simulation of different constructions and provides freedom for optimizations.

to have a rank assigned to certain states of the Büchi automaton. We therefore additionally store a separate "buffer set" in each macrostate with no assigned rank where such Büchi states can be placed in. The states in the buffer set are not used to track an accepting run directly, but they may reach successors later that should be managed inside of a tuple.

$$
\left(\left\{q_{1}, q_{3}\right\}^{5},\left\{q_{2}\right\}^{3}\right)\left|\left(\left\{q_{4}\right\}^{1},\left\{q_{6}\right\}^{6},\left\{q_{5}\right\}^{4}\right)\right|\left(\left\{q_{7}, q_{8}, q_{9}\right\}^{2}\right) \|\left\{q_{10}\right\}
$$

Fig. 2. Illustration of some macrostate of the modularized determinization procedure. In this example, assume that the underlying automaton has components with the states $q_{1}, q_{2}, q_{3}$ and $q_{4}, q_{5}, q_{6}$ and $q_{7}, \ldots, q_{10}$, respectively. The superscripts denote the global rank of the corresponding set. State $q_{10}$ is currently not considered to track accepting runs, so it is stored in the separated buffer set and has no assigned rank. The vertical bars separate the different components from each other.

To ensure the correct interaction of the tuples, the ranks assigned to sets must be unique in the whole macrostate. Apart from this difference, the construction above is applied to each tuple individually. Whenever a transition in the Büchi automaton moves from an SCC $C_{1}$ to a different SCC $C_{2}$, and the same state is not also reached by some transition inside of $C_{2}$, then the resulting state is added in a new right-most set in the tuple responsible for SCC $C_{2}$. The rank of this new set is the least important (i.e., largest in the tuple) one.

The correctness of this modular approach essentially follows from the fact that each accepting run eventually stays in the same SCC of the Büchi automaton forever, i.e., the states along this run eventually stay in the same tuple forever and are managed by the construction above without interference. More details about the required adaptations can be found in Appendix A.

\section{Optimizations and Heuristics}

In the following, we describe the optimizations and heuristics we suggest to consider during determinization of Büchi automata. 


\subsection{Using known language inclusions of Büchi states}

We are aware of only two rather simple optimizations that are exploiting known language inclusion relations between states of the Büchi automaton-a "trueloop" optimization from lt12dstar [20] that syntactically identifies NBA states that can be treated as accepting sinks, and an optimization used in spot [9] that is restricted to pairs of states from different SCCs of the NBA, which we refer to as "external" language inclusions. In general, it is nontrivial to use the language inclusions between pairs of states in the same SCC of the NBA, because both of them can occur infinitely often in the same run.

However, there is a possibility to use the language inclusion relation more generally within the same SCC in a safe manner by exploiting properties of the determinization construction from [25]. The result is what we call the "internal" language inclusion optimization, which works for the original non-modular version of the construction, which uses a single ranked tuple $(\alpha, t)$ per macrostate, as follows. Let $Q_{t}:=\bigcup_{i=1}^{n} S_{i}$ for $t=\left(S_{1}, \ldots, S_{n}\right)$ and let the function idx $x_{t}: Q_{t} \rightarrow[n]$ map each state $q \in Q_{t}$ to the tuple index $i$ such that $q \in S_{i}$.

Theorem 1. Let $p, q \in Q$ with $L(p) \subseteq L(q)$ and let $(\alpha, t)$ be a macrostate of the determinization construction such that $p, q \in Q_{t}$. If $q$ is to the left of $p$ (i.e. $\left.\operatorname{idx}_{t}(q)<\mathrm{idx}_{t}(p)\right)$, then $p$ may be omitted from the macrostate without changing the language of the determinized automaton.

A sketch of the correctness proof and required modifications for the modularized construction can be found in Appendix B.

Since the internal or external language inclusion optimization change the structure of the macrostates, the overall structure of the constructed DPA might change significantly. For this reason, there is no guarantee that this technique cannot increase the number of states in some cases. In our experiments we found no instance where this is the case, i.e., in practice they only decrease the number of states.

\subsection{Using properties of SCCs in the NBA}

In the following, we describe some heuristics to simplify the treatment of SCCs in the NBA that are rejecting, accepting, or deterministic. Earlier, we described how the determinization construction can be performed in a modular way. The following heuristics can be implemented very cleanly in that framework, but in principle could also be used with other constructions.

Rejecting SCCs: It is known that one can keep states from rejecting NBA SCCs separate from the determinization construction, as no accepting run can visit them infinitely often. This can be realized in our modular construction by keeping states of rejecting SCCs outside of the determinization tuples in the buffer set. A related optimization is already implemented in spot as a modification of the construction from [34]. 
Accepting SCCs: For accepting SCCs, it is sufficient to check that at least one run eventually stays in the same SCC forever. For this, an adaptation of the Miyano-Hayashi construction [29] (often called "breakpoint"-construction) can be used, which requires to manage only two different sets - a track set and a background set. In a transition, the track set is updated to all successors of the current track set that are in an accepting SCC. The background set contains all other states from accepting SCCs that are reached in this transition. This pair of sets has one rank assigned by the global ranking function. As long as the track set is non-empty, the rank signals a good event. If the track set becomes empty in a transition, then the rank signals a bad event, the background set becomes the new track set, and a new rank is assigned that is larger than all the ranks that survived the last transition. It is not very difficult to see that this construction correctly detects runs of the NBA that remain inside an accepting SCC.

This heuristic is realized in our modular construction by delaying the movement of states into the corresponding component tuple until a "breakpoint" happens, thereby ensuring that the tuple responsible for all accepting components always contains at most one non-empty set. If the two heuristics for rejecting and accepting SCCs are used, and the input NBA is a weak automaton (in which all SCCs are either rejecting or accepting), then one obtains the pure breakpoint construction, which is used, e.g., in [3] to determinize weak automata. The overall state complexity is then reduced from $2^{\mathcal{O}(n \log n)}$ to $3^{n}$. More details can be found in Appendix C.

Deterministic SCCs: If an SCC has both accepting and rejecting states, but is deterministic, a run never branches into multiple runs as long as the successors stay in the same component. Hence, the number of different runs can only decrease or stay the same. This excludes the possibility that an accepting state is visited infinitely often by different runs, but not by a single infinite run. Therefore, whenever a set of states in a deterministic SCC is reached from some other SCC, it suffices to add the states to the tuple which is responsible for this SCC with a new rank, but in the following steps there is no need to refine this set, i.e. separate accepting from non-accepting states, as this is only required for filtering out infinite non-accepting runs. For a good event to be signalled by such a component in the construction, it suffices that a set just contains an accepting state. If this set eventually never becomes empty and infinitely often contains accepting states, clearly at least one of the finitely many runs evolving in this set must visit accepting states infinitely often. Applying this heuristic on limitdeterministic NBA simplifies the determinization procedure to a variant of the construction described in [10], because then the importance ordering given by ranks coincides with the tuple index ordering, effectively mimicking the tuples as used in [10]. More details can be found in Appendix D.

It should be pointed out that all these SCC-based heuristics can in fact increase the number of states for specific instances, even though they restrict the state space in general (e.g. see Appendix F, Figure 6) and therefore must be applied with greater care. 


\subsection{Smart successor selection}

The determinization construction in [25] permits, in general, multiple valid successor macrostates because of the freedom in the merge operation. Determinization in practice usually works by starting in some initial state and fully exploring the state space which is reachable using the transitions prescribed by some construction. The natural optimization to be derived from the non-determinism of merge is to check for each transition whether a permissible successor state has already been constructed. In this case, just a new edge is added, and a new state is constructed only if no viable existing successor could be found.

The question is then how to find such a state as efficiently as possible. A naive approach would be to check for every already visited state whether it satisfies the constraints on the shape of a successor for the current transition. This would incur a blow-up in the running time that is quadratic in the size of the output automaton, which can be very large in general. A better approach is to structure the set of already visited states in a reasonable way that accelerates this search. We achieve this by managing a trie where each node corresponds to a macrostate. Each trie node is labelled by a set $S \subseteq Q$ of states of the NBA and can be marked to denote whether the corresponding macrostate already exists in the DPA. There exists a simple bijection that maps each ranked slice into a sequence of sets (without ranks) which uniquely determines a node in the trie. The resulting sequence of sets $S_{1}, S_{2}, \ldots, S_{n}$ is the "word" over the alphabet $2^{Q}$ which is inserted into the trie if the corresponding macrostate is added to the DPA (the sequence $S_{1}, S_{2}, \ldots, S_{n}$ is different from the tuple in the ranked slice).

When constructing a successor state, we first apply the operations step and prune. From that, we obtain the minimal active rank $k$. Under the constraints of the construction sketched in Section 3, the merge operation can now merge sets with rank at least $k$ which in turn means that the sets with rank smaller than $k$ do not change during merge. This sequence of sets of rank smaller than $k$ determines a trie node such that all possible successors that can be constructed by merge are below this node.

Only a simple check must be performed on remaining candidate macrostates that are found in the trie, and all required steps can be carried out efficiently using bit-set operations, so that the overall slowdown incurred by this optimized successor selection is very moderate. More details about this optimization can be found in Appendix E.

This optimization only prevents new redundant states from being constructed, thus the resulting automaton can never become larger than without this optimization enabled. However, in combination with other heuristics, like the postprocessing described in Section 4.5, it might also have a negative effect.

\subsection{The benefits of the powerset structure}

It is a well-known fact that language equivalent states of a deterministic or non-deterministic $\omega$-automaton cannot be merged, in general, without changing the accepted language. However, if the two states are in different SCCs, it is possible to remove one of them. The aim of the heuristic that we describe next, 
is to exploit this fact during construction of the DPA. Formally, we rely on the following proposition, which we consider folklore.

Proposition 1. Let $s, s^{\prime}$ be states of a DPA. If $L(s)=L\left(s^{\prime}\right)$ and $s \not \rightarrow s^{\prime}$, then all incoming edges of $s^{\prime}$ can be redirected to $s$ without changing the recognized language (and $s^{\prime}$ can be removed, since it becomes unreachable).

This implies that the whole SCC containing such a state $s^{\prime}$ can be removed because the language equivalence holds for all the successor states of $s$ and $s^{\prime}$ as well, due to the deterministic transitions.

In order to use this insight in the determinization construction, we need to detect language equivalences of states of the DPA at construction time. We do this by using the powerset structure $\operatorname{PS}(\mathcal{A})$ of the given NBA $\mathcal{A}$, which is the transition system with nodes from $2^{Q}$ obtained by using the NFA powerset construction on the Büchi automaton, with $Q_{0}$ as initial state and a deterministic transition function $\delta^{\mathrm{PS}(\mathcal{A})}(P, a):=\Delta(P, a)$. Our optimization is based on the following simple observation.

Proposition 2. Let $\mathcal{B}$ be a DPA that is equivalent to the $N B A \mathcal{A}$. Let $u$ be a finite word such that in $\mathcal{B}$ the state $s$ is reached via $u$, and in $\operatorname{PS}(\mathcal{A})$ the set $P$ is reached via $u$. Then $\mathcal{B}$ accepts from s the same language as $\mathcal{A}$ accepts from $P$.

This implies that states of the DPA that can be reached simultaneously with the same set $P$ in $\operatorname{PS}(\mathcal{A})$ are language equivalent. In combination with Proposition 1, we obtain that a DPA equivalent to $\mathcal{A}$ only needs one SCC per SCC in $\operatorname{PS}(\mathcal{A})$.

Corollary 1. Let $\mathcal{A}$ be an NBA and $\mathrm{PS}(\mathcal{A})$ its powerset structure. Then there exists a DPA $\mathcal{B}$ recognizing the same language such that for each $S C C$ of $\operatorname{PS}(\mathcal{A})$ there is at most one $S C C$ in $\mathcal{B}$.

Based on this observation, we separately construct for each $\operatorname{SCC} C$ of $\operatorname{PS}(\mathcal{A})$ one SCC of the DPA, as explained in the following. The construction picks some node $S \in C$ (which is a set of NBA states), and starts the determinization procedure using $S$ as initial states. Furthermore, it tracks for each macrostate $s$ also the corresponding node $P_{s}$ in $C$. On each transition, the successor macrostate for the DPA is only constructed, if the corresponding transition in $\operatorname{PS}(\mathcal{A})$ stays in $C$.

This construction gives us a DPA $\mathcal{B}_{C}$ that can be partial in the case of nonbottom SCCs $C$ of $\operatorname{PS}(\mathcal{A})$, with "holes" for transitions that exit $C$. Note that $\mathcal{B}_{C}$ might consist of several SCCs itself. Based on Propositions 1 and 2, we only need to keep one bottom SCC of $\mathcal{B}_{C}$.

In order to complete the missing transitions, consider such a transition leading outside of $C$ to some $P^{\prime}$ in an $\operatorname{SCC} C^{\prime}$ of $\operatorname{PS}(\mathcal{A})$. We assume that we have already done the determinization for $C^{\prime}$ (starting at the bottom $\operatorname{SCCs}$ of $\operatorname{PS}(\mathcal{A})$ and then going backwards), so there already exists a macrostate that corresponds to $P^{\prime}$, and we can let the transition of the DPA point to that macrostate. This idea is illustrated in Figure 3.

Putting this into practice and keeping only bottom SCCs of the partial DPAs that have the smallest size, is what we call the "topological" optimization. While 


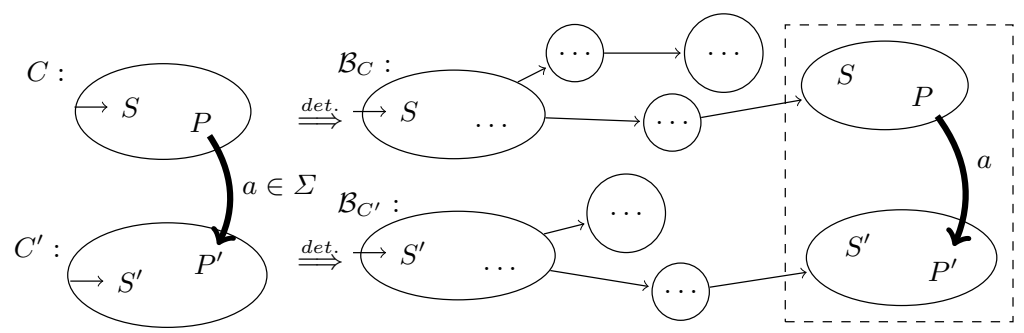

Fig. 3. Abstract sketch of the determinization guided by SCCs of $\operatorname{PS}(\mathcal{A})$. The two powerset SCCs $C$ and $C^{\prime}$ were determinized starting with the sets $S$ and $S^{\prime}$, respectively. The states in the constructed partial DPAs $\mathcal{B}_{C}$ and $\mathcal{B}_{C^{\prime}}$ are depicted by the sets from $\operatorname{PS}(\mathcal{A})$ to which they are language-equivalent. It suffices to keep just one bottom SCC of each partial DPA (e.g. in the dotted rectangle) and connect them by exploiting the known language equivalences to introduce missing edges (e.g. the depicted bold edge).

this still requires the exploration of all macrostates that are reached by the determinization of a single SCC in PS $(A)$, many of those may be removed afterwards.

Alternatively, one could explore in a depth-first fashion and greedily just keep the first completed bottom SCC, effectively trading an even smaller automaton size for possibly faster computation.

It can be easily implemented in such a way that the resulting automaton will have at most the same size as without this optimization, by picking appropriate initial states for the construction of the partial DPAs $\mathcal{B}_{C}$ (i.e. picking initial states that would also be reached by the unoptimized construction anyway).

Notice that this optimization is generic and can be used with any determinization construction based on state exploration.

\subsection{State reduction using Mealy minimization}

A simple but powerful optimization that to our knowledge has not been considered yet, consists of first minimizing the number of priorities of the DPA (which can be done efficiently using a simple algorithm from [5]) and then applying the classical minimization algorithm by Hopcroft [17], by interpreting the DPA as a Mealy machine that outputs priorities. Since the minimization as Mealy machine preserves the priority sequence for each input, this clearly does not change the language of the DPA.

We believe that the effectiveness is due to the determinization procedure often returning automata with an unnecessarily large number of different priorities. Thus, the new priority function may assign edges with initially distinct priorities the same new priority. Thereby, paths with initially different priority sequences might become equivalent, ultimately leading to more states that can be merged. Because this is a pure post-processing step, it can be integrated easily into any determinization tool-chain. 


\section{Experiments and Discussion}

Our quantitative experiments ${ }^{1}$ involved different sets of Büchi automata that can be roughly grouped into two categories - automata obtained from LTL formulas and arbitrary automata. Most sets were generated and/or processed using tools included with spot [9] (version 2.6.3). All sets were filtered to exclude already deterministic Büchi automata. Furthermore, we filtered the sets to include only automata that are determinizable by spot (via autfilt -D -P --high) in reasonable time (between 1-30 minutes, depending on the size of the corresponding test set). This was also our benchmark for comparison. We explicitly compare only the size of the resulting automata and do not aim for competitive performance. However, most heuristics we proposed are computationally cheap, being essentially simple modifications of the successor calculation.

All LTL formulas were transformed to state-based Büchi automata using ltl2tgba -B. As we are evaluating only the impact of the heuristics during the transformation from Büchi to parity automata, the method to transform LTL formulas to Büchi automata does not matter. For the same reason, we do not compare with tools like Rabinizer [22] that bypass Büchi automata and Safrastyle constructions. We are also aware of the fact that spot might have been able to produce smaller automata from LTL when using the usually smaller transition-based automata as input. As all our heuristics and optimizations in principle would work on those as well, we believe that this does not matter for our evaluation.

We used autcross to obtain statistics about different combinations of our heuristics as well as to ensure correctness of the generated automata. The test sets that we used were the following:

raut: 1662 random automata generated by randaut with 5-20 states (9 on average), mostly in one SCC.

rautms: 2112 random automata with 10-30 states (13 on average) generated by randaut, but filtered to have more than one SCC, specifically having at least one rejecting and at least one accepting component.

rltl: 11798 automata of various size ( 8 states on average) from random LTL formulas generated by randltl.

gltl: 68 automata from various parametrized LTL formula families generated by genltl that yield nondeterministic automata $[15,24,16,23,37,8,30]$.

scomp: 113 automata from LTL formulas extracted from the TLSF-benchmarks directory of the SYNTCOMP [18] benchmark repository.

To reduce the exponential number of possible combinations of different heuristics and algorithm variants to a manageable amount, we fixed an order in which to add optimizations additionally to the previous configuration. We tested this sequence of cumulatively enabled optimizations for the three successor construction strategies proposed in the description of the generalized determinization algorithm [25], which correspond to the Muller-Schupp construction (abbreviated

\footnotetext{
${ }^{1}$ Our prototype can be obtained at https://github.com/apirogov/nbautils
} 
M.-S.), Safra's construction and the maximally collapsing merge rule (abbreviated Max.). The following list explains the used parameter combinations. In the tables, the optimization listed in a column is enabled in addition to the ones used in all previous columns.

def: only trimming of the automaton and the known true-loop optimization [20]

$+\mathbf{T}$ : topological optimization (Sec. 4.4)

+E: known external language inclusion [9] (Sec. 4.1)

$+\mathbf{I}$ : internal language inclusion (Sec. 4.1)

$+\mathbf{M}$ : priority minimization and state reduction (Sec. 4.5$)$

$+\mathbf{S}$ : smart successor selection using tries (Sec. 4.3)

$+\mathbf{A}$ : breakpoint construction for accepting SCCs (Sec. 4.2)

$+\mathbf{W}$ : optimization for both, accepting and rejecting SCCs (Sec. 4.2)

+ D: optimization for deterministic SCCs (Sec. 4.2)

The results for our main benchmark test sets are shown in Table 1.

\begin{tabular}{r|l|l|llllllll} 
& $\Sigma$ spot & mode & def & $+\mathrm{T}$ & $+\mathrm{E}$ & $+\mathrm{I}$ & $+\mathrm{M}$ & $+\mathrm{S}$ & $+\mathrm{W}$ & $+\mathrm{D}$ \\
\hline \multirow{3}{*}{ raut } & \multirow{3}{*}{334523} & M.-S. & 2.42 & 2.31 & 2.31 & 2.31 & 1.33 & 1.07 & 1.07 & 1.07 \\
& & Safra & 1.14 & 1.00 & 1.00 & 1.00 & $\mathbf{0 . 8 0}$ & 0.82 & 0.82 & 0.82 \\
& & Max. & 1.21 & 1.07 & 1.06 & 1.06 & 0.84 & 0.83 & 0.83 & 0.83 \\
\hline \multirow{3}{*}{ rautms } & \multirow{3}{*}{286967} & M.-S. & 1.28 & 1.21 & 0.92 & 0.92 & 0.77 & 0.70 & 0.68 & 0.68 \\
& & Safra & 0.70 & 0.70 & 0.68 & 0.68 & 0.62 & 0.61 & $\mathbf{0 . 6 0}$ & 0.60 \\
& & Max. & 0.73 & 0.72 & 0.71 & 0.71 & 0.64 & 0.62 & 0.61 & 0.61 \\
\hline \multirow{3}{*}{ gltl } & \multirow{3}{*}{365251} & M.-S. & 3.63 & 2.05 & 1.42 & 1.33 & $\mathbf{0 . 8 4}$ & 0.93 & 0.94 & 0.90 \\
& & Safra & 4.15 & 2.67 & 1.76 & 1.61 & 0.93 & 0.96 & 0.97 & 0.92 \\
& & Max. & 3.94 & 2.47 & 1.79 & 1.65 & 0.98 & 0.99 & 1.01 & 0.93 \\
\hline \multirow{3}{*}{ scomp } & \multirow{3}{*}{14502} & M.-S. & 3.12 & 1.98 & 1.97 & 1.87 & 1.18 & 1.08 & 1.07 & 1.00 \\
& & Safra & 2.77 & 1.93 & 1.92 & 1.84 & 1.18 & 1.07 & 1.06 & 0.995 \\
& & Max. & 2.39 & 1.71 & 1.70 & 1.62 & 1.00 & 1.05 & 1.05 & $\mathbf{0 . 9 9 4}$ \\
\hline & & M.-S. & & & 1.84 & 1.59 & 0.97 & 0.77 & 0.85 & 0.74 \\
& & Safra & & & 1.92 & 1.58 & 0.98 & 0.72 & 0.78 & $\mathbf{0 . 6 9}$ \\
& & Max. & & & 1.62 & 1.22 & 0.73 & 0.74 & 0.85 & 0.70
\end{tabular}

Table 1. Results of the quantitative experiments. The numbers show the fraction of the sum of states obtained by the indicated configuration of our implementation, compared to the sum of states of the automata obtained by spot. On the SYNTCOMP set we did not evaluate the least optimized configurations, as some optimizations were necessary to obtain results in acceptable time.

Our results show that for each test set there is a configuration of our prototype that, on average, produces automata that are not larger than the ones produced by spot. In many cases, the best configuration produces automata with up to $40 \%$ less states. 
Unfortunately, the effect of the post-processing step with the priority minimization and state reduction $(+\mathbf{M})$ is not robust even under minor variations of the automaton. This is, e.g., witnessed by the test set rltl, for which the state reduction without the smart successor selection yields better results. As only states that agree on all priority sequences of runs going through them can be merged by Hopcroft's algorithm, slight variations that lead to "symmetry breaking" may already render states non-mergeable. This optimization is more costly than the others, because equivalent states must be computed in the resulting automaton. But we believe that this is one of the most generally useful optimizations which is worth the additional computation time, as it quite consistently reduces the number of states by approximately $20-40 \%$.

The smart selection of successors $(+\mathbf{S})$ is the only proposed optimization which is "stateful", in the sense that it depends on the already constructed part of the automaton, which in turn depends on other enabled optimizations. In all but one test sets, this optimization was especially helpful in reducing states when using the Muller-Schupp successors. Interestingly, it was most successful in the Safra-based construction for the SYNTCOMP test set, where this optimization alone was responsible for an additional state reduction of $26 \%$. The topological optimization $(+\mathbf{T})$ yields a mild state reduction on random automata and significant reduction of up to $36 \%$ in the LTL-based test sets. A particularly suggestive demonstration of the topological optimization is given in Appendix F, Figure 7.

The application of the optimization that exploits language inclusion relations inside of a single SCC $(+\mathbf{I})$ seems to have no visible effect on random automata, but was responsible for a decent additional state reduction of $6-8 \%$ for any variant of the construction in the LTL-based test sets. In our prototype we used only a simple direct simulation [11] for the optimizations. We are optimistic that using more involved simulations [12] to under-approximate language inclusion, e.g. fair simulation, would lead to even better results.

The heuristics for accepting and rejecting SCCs of the NBA $(+\mathbf{W})$, while sometimes helpful, in other cases lead to an increase of the number of states. The separate handling of deterministic components, which is enabled in addition to all other optimizations, shows a positive effect mainly in the LTL-based automata test sets.

We now give some further examples that illustrate the potential effect of some of the heuristics. We start with the effect of our state reduction when determinizing the family of automata introduced by Max Michel in [28] (see also [39]) and which was used as a benchmark in [1]. A deterministic automaton recognizing the same language must have at least $n$ ! states and is usually much larger in practice. While in [1] the Muller-Schupp construction performed significantly worse than Safra's construction, we were surprised to see that using the Muller-Schupp update in our own experiments, the Hopcroft minimization is able to drastically reduce the size of the automaton, producing much smaller automata than any other construction variant we tried (see Table 2). 


\begin{tabular}{r|r|l|rr||r|r|r|rr}
$n$ & spot & mode & def + TEI & + M & $n$ & spot & mode & def + TEI & + M \\
\hline 2 & 18 & M.-S. & 19 & $\mathbf{1 7}$ & 4 & 2284 & M.-S. & 2725 & $\mathbf{4 5 7}$ \\
& & Safra & 18 & 18 & & & Safra & 2202 & 2106 \\
& & Max. & 18 & 18 & & & Max. & 2094 & 2094 \\
\hline 3 & 145 & M.-S. & 166 & $\mathbf{8 2}$ & 5 & 60109 & M.-S. & 72616 & $\mathbf{2 9 3 6}$ \\
& & Safra & 142 & 142 & & & Safra & 57714 & 57714 \\
& & Max. & 142 & 142 & & & Max. & 51094 & 51094
\end{tabular}

Table 2. Results for Michel's automata family (input NBA have $n+1$ states for all $n$ ). The table demonstrates the surprising efficiency of the Muller-Schupp construction with subsequent state reduction.

The heuristics for handling rejecting/accepting $(+\mathbf{W})$ and deterministic $(+\mathbf{D})$ SCCs of the NBA were enabled in addition to all other heuristics in the experiments shown in Table 1, and thus only had a small or even negative effect. We refer to Appendix F, Figure 6 for a concrete example where SCC-based heuristics can be harmful. In the following we give some examples showing that these heuristics indeed can have a strong positive effect. The deterministic SCC optimization is very useful for determinization of automata obtained from formulas $\varphi_{G H}(n):=\bigwedge_{i=1}^{n} \mathrm{GF} a_{i} \vee \mathrm{FG} a_{i+1}$ from [16]. An example where the heuristic which applies the breakpoint-style construction to accepting SCCs is very helpful is the family of formulas $\varphi_{M S}(n):=\bigvee_{i=0}^{n} \mathrm{FG}\left(\neg^{i} a \vee \mathrm{X}^{i} b\right)$ from [30], where the bottom SCCs of the automata are increasingly complex accepting SCCs (see Table 3).

\begin{tabular}{c|r|rr|rr|rr} 
& & M.-S. & Safra & max. \\
\hline formula & spot & def.+TEI & $+\mathrm{D}$ & def.+TEI & $+\mathrm{D}$ & def.+TEI & $+\mathrm{D}$ \\
\hline$\varphi_{G H}(2)$ & 18 & 30 & 32 & 29 & 32 & 28 & 32 \\
$\varphi_{G H}(3)$ & 108 & 385 & 255 & 327 & 255 & 381 & 266 \\
$\varphi_{G H}(4)$ & 2143 & 15206 & 5612 & 10922 & 5612 & 12394 & 5036 \\
\hline formula & spot & def.+TEI & $+\mathrm{A}$ & def.+TEI & $+\mathrm{A}$ & def.+TEI & $+\mathrm{A}$ \\
\hline$\varphi_{M S}(2)$ & 21 & 40 & 16 & 40 & 16 & 36 & 16 \\
$\varphi_{M S}(3)$ & 170 & 371 & 46 & 371 & 46 & 155 & 46 \\
$\varphi_{M S}(4)$ & 1816 & 4933 & 132 & 4933 & 132 & 620 & 132 \\
$\varphi_{M S}(5)$ & 22196 & 67173 & 358 & 67173 & 358 & 2419 & 358
\end{tabular}

Table 3. Results for some instances of the $\varphi_{G H}(n)$ and $\varphi_{M S}(n)$ formulas, with and without usage of suitable heuristics. Number of states obtained by spot is provided as reference in the tables. In case of $\varphi_{G H}$ formulas, spot profits significantly from the stutter-invariance optimization [21], which we do not utilize.

In summary, we noticed that the positive effects of the heuristics become stronger with growing size of the input automaton. This is not surprising, as for e.g. the SCC heuristics to have a positive effect, the automaton needs to be sufficiently complex. Unfortunately, larger input automata are not suitable for a thorough quantitative analysis in reasonable time. We are convinced, that for sufficiently large automata, even the heuristics that may appear not very 
effective in the presented benchmarks would have a stronger positive effect on average.

Our results also show that every proposed choice of the merge operation in the unified determinization construction seems to be superior in some cases. The Muller-Schupp update was the best choice on the random LTL test set, while the maximal collapse update was superior on the test set with the parametrized LTL formulas. Even though the unoptimized Muller-Schupp update usually seems to perform worst, combining it with some optimizations makes it a viable choice. The maximal collapsing update seems to perform comparably with the Safra update, but appears to be slightly worse in most cases, whereas the well-known Safra update seems to be a good middle ground.

\section{Conclusion}

We presented a number of new heuristic optimizations for the determinization of Büchi automata, and evaluated them in a prototype implementation using different test sets of automata, ranging from randomly generated automata to automata constructed from specifications from the competition SYNTCOMP. Our results show that these heuristics can significantly reduce the number of states in comparison with the base construction, and also in comparison with the current state-of-the-art tool spot for determinization of NBAs.

In future work, we want to study in more depth the effect of the heuristic based on language inclusions, by using stronger tools for identifying language inclusions between states of the Büchi automaton (currently we are only using direct simulation). We also see further potential in the smart successor selection, which could be used for redirecting transitions in the constructed automaton in order to reduce its size.

\section{References}

1. Althoff, C.S., Thomas, W., Wallmeier, N.: Observations on determinization of Büchi automata. In: CIAA 2005. pp. 262-272. Springer

2. Baier, C., Katoen, J.P.: Principles of model checking. MIT Press (2008)

3. Boigelot, B., Jodogne, S., Wolper, P.: On the use of weak automata for deciding linear arithmetic with integer and real variables. In: IJCAR 2001. Springer

4. Büchi, J.R.: On a decision method in restricted second order arithmetic. In: Studies in Logic and the Foundations of Mathematics, vol. 44, pp. 1-11. Elsevier (1966)

5. Carton, O., Maceiras, R.: Computing the Rabin index of a parity automaton. RAIRO-Theoretical Informatics and Applications 33(6), 495-505 (1999)

6. Choffrut, C., Grigorieff, S.: Uniformization of rational relations. In: Jewels are Forever. pp. 59-71. Springer (1999)

7. Colcombet, T., Zdanowski, K.: A tight lower bound for determinization of transition labeled Büchi automata. In: ICALP 2009. Springer

8. Duret-Lutz, A.: Manipulating LTL formulas using spot 1.0. In: ATVA 2013. Springer

9. Duret-Lutz, A., Lewkowicz, A., Fauchille, A., Michaud, T., Renault, E., Xu, L.: Spot 2.0-a framework for LTL and $\omega$-automata manipulation. In: ATVA 2016. Springer 
10. Esparza, J., Křetínskỳ, J., Raskin, J.F., Sickert, S.: From LTL and limitdeterministic Büchi automata to deterministic parity automata. In: TACAS 2017. Springer

11. Etessami, K., Holzmann, G.J.: Optimizing Büchi automata. In: CONCUR 2000. Springer

12. Etessami, K., Wilke, T., Schuller, R.A.: Fair simulation relations, parity games, and state space reduction for Büchi automata. SIAM J. on Computing 34(5) (2005)

13. Fisman, D., Lustig, Y.: A modular approach for Büchi determinization. In: CONCUR 2015. LIPICs

14. Fogarty, S., Kupferman, O., Vardi, M.Y., Wilke, T.: Profile trees for Büchi word automata, with application to determinization. Information and Computation 245, 136-151 (2015)

15. Gastin, P., Oddoux, D.: Fast LTL to Büchi automata translation. In: CAV 2001. Springer

16. Geldenhuys, J., Hansen, H.: Larger automata and less work for LTL model checking. In: SPIN 2006. Springer

17. Hopcroft, J.: An $\mathrm{n} \log \mathrm{n}$ algorithm for minimizing states in a finite automaton. In: Theory of machines and computations, pp. 189-196. Elsevier (1971)

18. Jacobs, S., Basset, N., Bloem, R., Brenguier, R., Colange, M., Faymonville, P., Finkbeiner, B., Khalimov, A., Klein, F., Michaud, T., et al.: The 4th reactive synthesis competition (syntcomp 2017): Benchmarks, participants \& results. arXiv preprint arXiv:1711.11439 (2017)

19. Kähler, D., Wilke, T.: Complementation, disambiguation, and determinization of Büchi automata unified. In: ICALP 2008. Springer

20. Klein, J.: Linear time logic and deterministic omega-automata. Diploma thesis, University of Bonn (2005)

21. Klein, J., Baier, C.: On-the-fly stuttering in the construction of deterministic omega-automata. In: CIAA 2007. Springer

22. Křetínskỳ, J., Meggendorfer, T., Sickert, S., Ziegler, C.: Rabinizer 4: from LTL to your favourite deterministic automaton. In: CAV 2018. Springer

23. Kupferman, O., Rosenberg, A.: The blow-up in translating LTL to deterministic automata. In: MoChArt 2010. Springer

24. Kupferman, O., Vardi, M.Y.: From linear time to branching time. TOCL 2005

25. Löding, C., Pirogov, A.: Determinization of Büchi automata: Unifying the approaches of Safra and Muller-Schupp. ICALP 2019 (to appear) https://arxiv.org/abs/1902.02139

26. McNaughton, R.: Testing and generating infinite sequences by a finite automaton. Information and control 9(5), 521-530 (1966)

27. Meyer, P.J., Sickert, S., Luttenberger, M.: Strix: Explicit reactive synthesis strikes back! In: CAV 2018. Springer

28. Michel, M.: Complementation is more difficult with automata on infinite words. Manuscript, CNET, Paris (1988)

29. Miyano, S., Hayashi, T.: Alternating finite automata on $\omega$-words. Theoretical Computer Science 32(3), 321-330 (1984)

30. Müller, D., Sickert, S.: LTL to deterministic Emerson-Lei automata. In: GandALF 2017

31. Muller, D.E., Schupp, P.E.: Simulating alternating tree automata by nondeterministic automata: New results and new proofs of the theorems of Rabin, McNaughton and Safra. Theoretical Computer Science 141(1-2), 69-107 (1995)

32. Piterman, N.: From nondeterministic Büchi and Streett automata to deterministic parity automata. In: LICS 2006. IEEE 
33. Pnueli, A.: The temporal logic of programs. In: Foundations of Computer Science, 1977., 18th Annual Symposium on. pp. 46-57. IEEE (1977)

34. Redziejowski, R.R.: An improved construction of deterministic omega-automaton using derivatives. Fundamenta Informaticae 119(3-4), 393-406 (2012)

35. Safra, S.: On the complexity of omega-automata. In: Foundations of Computer Science, 1988., 29th Annual Symposium on. pp. 319-327. IEEE (1988)

36. Schewe, S.: Tighter bounds for the determinisation of Büchi automata. In: FOSSACS 2009. Springer

37. Tabakov, D., Vardi, M.Y.: Optimized temporal monitors for SystemC. In: International Conference on Runtime Verification. pp. 436-451. Springer (2010)

38. Thomas, W.: Automata on infinite objects. In: Handbook of Theoretical Computer Science, vol. B, pp. 133-192. Elsevier Science Publishers, Amsterdam (1990)

39. Thomas, W.: Handbook of formal languages, vol. 3. chap. Languages, Automata, and Logic, pp. 389-455. Springer (1997)

40. Thomas, W.: Church's problem and a tour through automata theory. In: Pillars of Computer Science. pp. 635-655. Springer (2008)

41. Vardi, M.Y., Wilke, T.: Automata: from logics to algorithms. In: Logic and automata - history and perspectives, Texts in Logic and Games, vol. 2, pp. 629-724. Amsterdam University Press (2007) 


\section{A Adaptations for the modularized construction}

In this section, we provide some more details on our modularized determinization construction that is based on the construction presented in [25] and was sketched in Section 3.1.

Fix a Büchi automaton $\mathcal{A}=\left(\Sigma, Q, \Delta, Q_{0}, F\right)$ and observe that each accepting run of the Büchi automaton must eventually stay in the same SCC of $\mathcal{A}$ forever. Furthermore, a run can switch between components only a finite number of times. So one can run multiple instances of the determinization in parallel, each responsible for one (or multiple) of the SCCs of the Büchi automaton, and try to detect accepting runs that eventually stay in those components.

Formally, let $P=\left\{P_{i}\right\}_{i=1}^{|P|}$ be an SCC-preserving partition of $Q$, where SCCpreserving means that for $p, q \in Q, \operatorname{scc}(p)=\operatorname{scc}(q)$ implies that $p, q \in P_{i}$ for the same $i \in \mathbb{N}$. Fix $m \in \mathbb{N}$, denoting the number of determinization tuples that will be run in parallel, and choose an injective map prt : $[m] \hookrightarrow P$ that assigns each tuple a different set of states from the Büchi automaton that it will be responsible for. The assignment should cover all SCCs of the Büchi automaton which admit accepting runs, i.e. $Q \backslash R \subseteq \bigcup_{i=1}^{m} \operatorname{prt}(i)$, where $R$ is the set of states in rejecting $\mathrm{SCCs}$ of $\mathcal{A}$.

A macrostate $s:=\left(\left(\alpha_{i}, t_{i}\right)_{i=1}^{m}, B\right)$ in the modular construction consists of a buffer set $B \subseteq Q$ and components $\left(\alpha_{i}, t_{i}\right)$ with tuples $t_{i}:=\left(S_{i, 1}, \ldots, S_{i, n_{i}}\right)$ equipped with injective functions $\alpha_{i}:\left[n_{i}\right] \hookrightarrow[n]$ that map each set to its rank, where $n:=\sum_{i=1}^{m} n_{i}, Q_{t_{i}}:=\bigcup_{j=1}^{n_{i}} S_{i, j}$ and $Q_{s}:=B \cup \bigcup_{i=1}^{m} Q_{t_{i}}$. We require that the sets $\operatorname{img}\left(\alpha_{i}\right)$ partition $[n], Q_{t_{i}} \subseteq \operatorname{prt}(i)$ and that all sets of states in $s$ must be disjoint. We allow the components to be empty, i.e. having $n_{i}=0$ (then $\alpha_{i}$ is just the empty function).

Whenever a state (of the NBA) is reached from some other state in the same component, we must follow the rules of the original procedure to infer accepting runs correctly. However, when a state is reached only from a state in a different component, then the state must lie on a run which is not already represented in the tuple for that component. Hence, we can add this state to the component in such a way that it is not related to the others and from there on let the original construction handle the rest.

The priorities at the transitions of the original construction are consequence of red and green ranks that are assigned to sets. When multiple components work in parallel and one component witnesses an accepting run by signalling green infinitely often (and red only finitely often), these signals must eventually dominate red signals from other components. The correct interaction of signals is easily achieved by using a ranking that totally orders all the sets (which is captured by the definition and constraints on partial rankings $\alpha_{i}$ ), disregarding the separated components, and updating the ranks according to the rules of the construction. Following the construction ensures that "less important" sets move up in the ranking order whenever a more important rank has a bad event.

The initial state consists of a macrostate $q_{0}$ such that all states in $Q_{0}$ are distributed into singleton sets in the corresponding tuples $t_{i}$, with arbitrary different ranks satisfying the definition of macrostates above. 
A transition is defined as follows. Let $s=\left(\left(\alpha_{i}, t_{i}\right)_{i=1}^{m}, B\right)$ be some macrostate. Similar to the original construction, the successor on some letter $x \in \Sigma$ is obtained by performing the steps step, relocate, prune, merge and normalize, where only the relocate step is completely new, whereas the other steps are only small modifications of the original construction.

First, the operation step is executed on each tuple exactly as before. Additionally, let $\tilde{B}:=\Delta(B, x)$ be the successors of states in the buffer set. The new operation relocate collects the set of all wrongly-placed states from the sets $\tilde{B}$ and $\tilde{Q}_{t_{i}}$, removes them from those sets and places them into the correct sets.

Formally, let $W_{0}:=\tilde{B} \cap \bigcup_{i=1}^{m} \operatorname{prt}(i)$ and for $1 \leq i \leq m$ let $W_{i}:=\left\{q \in Q_{\tilde{t}_{i}} \mid\right.$ $q \notin \operatorname{prt}(i)\}$. Then $\stackrel{\circ}{B}:=\left(\tilde{B} \backslash W_{0}\right) \cup\left(\left(\bigcup_{i=1}^{m} W_{i}\right) \backslash \bigcup_{i=1}^{m} \operatorname{prt}(i)\right)$ is the new buffer set, containing all reached states that do not belong in any tuple. The tuples $\dot{t}_{i}$ are defined by setting $\stackrel{\circ}{S}_{i, j}:=\tilde{S}_{i, j} \backslash W_{i}$ for each set $\tilde{S}_{i, j}$ in $\tilde{t}_{i}$, thereby removing wrongly placed states, and then adding a new rightmost set $\stackrel{\circ}{S}_{i, n e w}:=\left(\bigcup_{j=0}^{m} W_{j} \cap\right.$ $\operatorname{prt}(i)) \backslash Q_{\tilde{t}_{i}}$ to the tuple, which contains states that have predecessors only outside of the tuple. The ranks $\stackrel{\circ}{\alpha}$ for the modified sets stay the same as before, whereas

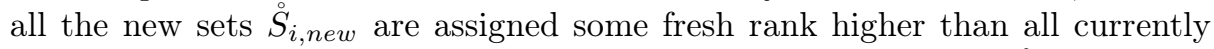
assigned ranks, giving us the intermediate macrostate $\left(\left(\stackrel{\circ}{\alpha}_{i}, \stackrel{\circ}{t}_{i}\right)_{i=1}^{m}, \stackrel{\circ}{B}\right)$.

Next, proceed with prune as in the original construction. The priority of the transition is obtained in essentially the same way as before, based on the results of prune in the tuples. First obtain the sets $G_{i}$ and $R_{i}$ of good and bad ranks of the different tuples according to the original construction, then consider their union $G:=\bigcup_{i=1}^{m} G_{i}$ and $R:=\bigcup_{i=1}^{m} R_{i}$. The priority $p$ of the transition is $2 k$ if $k \in G$ and $2 k-1$ otherwise, where $k:=\min (G \cup R$ ) (or $k:=|Q|+1$ if $(G \cup R)=\emptyset)$ denotes the smallest rank associated with some event in a tuple.

The merge operation has constraints based on the smallest rank with a good or bad event in the transition. Use the most important rank $k$ of the whole macrostate from above to execute merge on the individual tuples. Finally, apply normalize in such a way that the rank order of all sets in the whole macrostate is preserved and let $B^{\prime}:=\stackrel{\circ}{B}$, so that in the end we have the successor macrostate $s^{\prime}=\left(\left(\alpha_{i}^{\prime}, t_{i}^{\prime}\right)_{i=1}^{m}, B^{\prime}\right)$.

A note to readers familiar with Safra's construction - the redistribution of misplaced states in the relocate operation essentially introduces a new separate Safra tree root labelled by the newly introduced states. The tree structure here is encoded in the ranks (more details on this correspondence can be found in [25]). By adding the new rightmost sets $\stackrel{\circ}{i, n e w}_{i \text { as described above, each tuple in general }}$ contains not just one tree encoding the relationship of runs that share a common history, but multiple such trees in an ordered forest, reflecting the "unknown" history which was lost in the transition of the state between the different tuples. The important preserved information is that the state was actually reached at some time $i$ and from this point on the runs that continue from there and stay in the same component will be correctly managed like in the original construction. 


\section{B Correctness of internal language inclusion optimization}

In this section we give a proof sketch for the proposed internal language inclusion optimization from Section 4.1:

Theorem 1. Let $p, q \in Q$ with $L(p) \subseteq L(q)$ and let $(\alpha, t)$ be a macrostate of the determinization construction such that $p, q \in Q_{t}$. If $q$ is to the left of $p$ (i.e. $\left.\mathrm{idx}_{t}(q)<\mathrm{idx}_{t}(p)\right)$, then $p$ may be omitted from the macrostate without changing the language of the determinized automaton.

Proof (sketch). Let $w \in L(\mathcal{A})$. We need to show that removing states in this way does not inhibit the acceptance of $w$ by the resulting DPA. Let the acceptance profile of a run $q_{0} q_{1} \ldots$ be defined as a sequence $z_{0} z_{1} \ldots \in\{0,1\}^{\omega}$ such that $z_{i}=1$ if $q_{i} \in F$ and $z_{i}=0$ otherwise. Then let $\rho$ be an accepting run with a lexicographically maximal acceptance profile, i.e., an accepting run visiting accepting states as early as possible (such a run always exists, see e.g., the greedy ordering of runs in [6]).

The determinization construction puts states along lexicographically larger runs to the left of states along lexicographically smaller runs by separating accepting from non-accepting states and keeping only leftmost occurrences of each state in step. This order is never swapped during the other operations. It can only happen that two states are merged into the same set in merge. Thus, the construction ensures the following invariant: $\mathrm{idx}_{t}(q)<\mathrm{idx}_{t}(p)$ implies that a lex. maximal run going through $q$ is lex. larger than a lex. maximal run going through $p$ at the same time. Hence, in the sequence of macrostates on $w$ in a DPA, no state along the run $\rho$ is ever removed by this optimization, because this would contradict the maximality of the acceptance profile of $\rho$. Choosing this run $\rho$ in the proof of [25, Lemma 6] shows that the DPA indeed still accepts $w$.

\section{B.1 Modification for the modular construction}

The invariant in the proof above may be violated in the modular construction (Appendix A) due to states being moved between different tuples, such that some run which goes through these states may suddenly switch to the right side of a tuple, even though it is "better" than other runs tracked in that tuple. This poses a problem, if the same run can leave and re-enter the same tuple. Therefore, we need to add an additional constraint to pairs of states for which we can apply this optimization to exclude this possibility.

More formally, in the modular construction we may only apply Theorem 1 to some pair of NBA states $p, q \in Q$ with $L(p) \subseteq L(q)$ that are assigned to the same determinization tuple $t_{i}$ (i.e., $p, q \in \operatorname{prt}(i)$ for some $i \in \mathbb{N}$ ), if every path from $q$ to $p$ must stay in $\operatorname{prt}(i)$, i.e., can not leave and re-enter $t_{i}$.

\section{Details on the accepting SCC heuristic}

In this section we describe how the breakpoint construction [29] can be internalized in the determinization procedure to handle accepting SCCs of Büchi automata more efficiently. 
The idea of the breakpoint construction is that for accepting SCCs it is sufficient to check that at least one run eventually stays in the same SCC forever. Because there are no rejecting loops that could be taken by runs in these SCCs, it suffices to (conceptually) fix some set of runs that are currently in such SCCs and track sets of states that are reached by these runs and stay in those SCCs. As long as there is at least one state left, there must be a run remaining in this SCC and all remaining runs must be accepting. This reduces the required effort to track accepting runs significantly. One only needs to ensure the separation of the states in these accepting SCCs reached by the currently tracked runs from states that were reached only by runs that are currently not followed. When our currently tracked set becomes empty, we can start the tracking again on (a subset of) states along the previously unfollowed runs. If it is ensured that every reached state in the accepting SCCs eventually has the chance to be tracked, an accepting run will eventually be detected, if one exists.

So we need to manage only two different sets to detect accepting runs in any accepting SCC - a track set and a background set. In a transition, the track set is updated to all successors of the current track set that are in an accepting SCC. The background set contains all other states from accepting SCCs that are reached in this transition. Whenever the track set becomes empty (which is a "breakpoint"), all states from the background set are moved to the track set. Using this kind of scheme (see e.g. [3]), it is possible to determinize weak NBA with a blow-up of at most $3^{n}$, instead of the general upper bound of $2^{\mathcal{O}(n \log n)}$.

To exploit this approach for general NBA, we utilize our modular determinization construction (see Appendix A). We use the buffer set $B$ of the modular macrostates for the background set and a separate determinization component for the track set, which is formally some ranked tuple $\left(\alpha_{i}, t_{i}\right)$ with $t_{i}:=\left(S_{i, 1}\right)$ containing a single set with all the states in the track set. This component is responsible for all accepting SCCs, i.e., $\operatorname{prt}(i):=A$, where $A$ is the union of all accepting SCCs of the NBA. The operation relocate is modified in such a way that reached states in $A$ are collected in the buffer set $B$ first and $d o$ not go directly into the responsible component $\left(\alpha_{i}, t_{i}\right)$, unless it becomes empty during step (which indicates a breakpoint).

As all states in $A$ are accepting, they all move to the left child during step, whereas the right child becomes empty. Consequently, the right empty set is removed, whereas its rank (which is more important) is preserved by assigning it to the left child containing all the states. This rank, by definition, is in the set $G_{i}$, signalling a good event. On the other hand, if the tracked states have no successors, both child sets will be empty during step, resulting in prune removing both of them and the assigned rank (as well as the fresh rank of the left child set) signalling a bad event, i.e., being in the set $R_{i}$. But then all other states in $A$ that are currently untracked are moved into the component by relocate with a fresh rank of low importance, thereby "restarting" the track set with the states from the background set. The merge operation on a single set clearly is always trivial (i.e. can be skipped). 
In practice, the separation of successors into two sets during step and most of the mechanics during prune can be optimized away by making the current rank of the single set in the tuple always signal green, unless it becomes empty, when it must signal red. Thereby we can use only a single rank (assigned by $\alpha_{i}$ ) for all states in $A$. If the track set in the tuple eventually never becomes empty, its importance will increase by following the construction and eventually its green signal will be the most important in the transition, unless there exists a different more important rank that also eventually always signals green, so the DPA is guaranteed to accept if there is an accepting run in the accepting SCCs of the Büchi automaton. On the other hand, if there is no such run, then the determinization component that runs the breakpoint construction will become empty and will be "restarted" infinitely often and therefore its current rank will infinitely often signal red and thus will never witness an accepting run.

\section{Details on the deterministic SCC heuristic}

In this section we describe how the determinization construction can be simplified for SCCs of the NBA that are already deterministic. For this section, fix a deterministic SCC $D$ of the Büchi automaton. The idea is based on the fact that no run of the Büchi automaton that currently is in $D$ can ever split up into multiple runs as long as its states remain in $D$.

Fix (conceptually) a set of runs currently in $D$, i.e., take the set of states occupied by these runs. Then track for these states which successors also remain in $D$ (if a run splits up, then all but one successors go to a different SCC and are not tracked). Clearly, as long as the tracked set is not empty, there are still runs in $D$. Furthermore, if an accepting state is in this set, then it was reached by one of the runs we are currently tracking. Therefore, if there are accepting states in this set infinitely often, then at least one of the finitely many runs tracked in the set must visit accepting states infinitely often, i.e. there exists an accepting run.

While we are tracking this set, some runs from other SCCs may enter $D$ and reach the same or also different states. Whenever this happens, we start a new tracking set with a lower priority, which means that whenever both sets reach the same state $q$, only the set with higher priority gets state $q$. Thus, we keep only one copy of each state and at the same time lower priority sets never interfere with higher priority sets. Lower priority sets therefore only track runs that visit states which are not visited by runs tracked in sets with higher priority at the same time.

Notice how this concept can be easily represented in our modularized construction (see Appendix A). Assume that some determinization component $\left(\alpha_{i}, t_{i}\right)$ is responsible for the deterministic SCC $D$ of the NBA, i.e. we have that $\operatorname{prt}(i)=$ $D$. We can easily adapt the step operation to not split up the set of successors, so that together with the relocate operation we get exactly the behaviour described above, i.e., older sets are located to the left in $t_{i}$ and are favoured by the normalized successor calculation, whereas new sets are introduced on the right 
by relocate. These sets contain only states that were not reached by the other sets in the component and get a fresh rank with low importance. States that leave $D$ are also automatically taken care of by the construction during relocate. Whenever a tracked set becomes empty, it will be removed by prune and by definition, its rank will be in the set $R_{i}$ representing a bad event. For a rank to signal a good event, we modify the construction for this component to reflect the reasoning above, such that a rank $r$ is put into the set $G_{i}$ whenever the set $S_{i, j}$ in $t_{i}$ with $\alpha_{i}(j)=r$ contains an accepting state. The operations merge and normalize work as before.

Observe that by disabling the splitting of sets during step we can hope to construct less different partitionings of the states in $D$, as new sets only are introduced by runs coming from outside of $D$. Furthermore, the assigned ranks in the tuple $t_{i}$ are always ascending (because the new sets with low importance are always added on the right), so that the importance order and tuple order agree. This property restricts the worst-case upper bound on the state space for the component $\left(\alpha_{i}, t_{i}\right)$, as only ascending rankings $\alpha_{i}$ are used.

When applying this heuristic for the determinization of limit-deterministic automata, one obtains a variant of the construction described in [10], in which the states in the deterministic components are organized in a tuple sorted by the order in which the corresponding run entered the deterministic part. In our construction, we use a tuple of sets instead, tracking those runs that enter the deterministic part at the same time in one set. While the worst-case upper bound for the number of resulting macrostates is better for the construction in [10], in our experiments we found that using tuples of sets also works well in the examples. 


\section{E Details on smart successor selection}

In this section, we describe in more detail how the freedom of choice for the successor given by the merge operation of the determinization construction from [25] can be exploited in a computationally feasible way, i.e., how to quickly search for a candidate successor macrostate for some transition during state exploration of the DPA in the set of already existing macrostates.

The crucial idea is to structure the set of already visited states in a reasonable way such that macrostates which share a common prefix in the trie are related wrt. the tree interpretation of the macrostates. This tree is derived from a ranked slice in the following way. Let $(\alpha, t)$ be a ranked slice with $t=\left(S_{1}, \ldots, S_{n}\right)$. The tuple index of the parent of $S_{i}$ is the closest index to the right of $i$ that has a smaller rank, formally defined as $\uparrow(i):=\min _{i<k \leq n}\{k \mid \alpha(k)<\alpha(i)\}$. The ordered tree induced by $\uparrow$, with siblings in tuple index order, is called the ranktree of $(\alpha, t)$ (more on this correspondence of ranked slices and trees can be found in $[25])$.

For example, in the macrostate $\left(\left\{q_{3}, q_{4}\right\}^{4},\left\{q_{2}\right\}^{2},\left\{q_{5}, q_{6}\right\}^{3},\left\{q_{1}\right\}^{1}\right)$ from Figure 5(b), the rightmost index 4 can have no parent and is the root. Its rank 1 is the closest smaller rank for positions 2 and 3 (that have ranks 2 and 3, respectively), hence we have $\uparrow(2)=\uparrow(3)=4$, whereas the position 1 (with rank 4) has position 2 (with rank 2 ) as parent, that is $\uparrow(1)=2$. The edges shown in Figure 5(a)-(c) correspond to the edges in this tree interpretation, pointing from a parent to its direct descendants. In Figure 4, the trees on the bottom are actually such macrostates, in this interpretation as trees. The sequence of sets in the upper part of the figure is explained later.

Rank-trees have the property that parents have smaller ranks than children and sibling nodes have ascending ranks, which easily follows from the definition. So one can interpret ranks as the order of creation of nodes in the tree, smaller ranks meaning that the corresponding tree node was created earlier (in practice, multiple nodes that are created at the same time are eventually assigned different ranks during the normalize step of the determinization construction, fixing an arbitrary creation order for them).

We now explain how such rank trees can be encoded by a single sequence of sets (with ranks implicitly encoded), which is the basis for the data structure that we are using. We present it here for the case of a single tuple, but it can be adapted to the modularized variant in a straight-forward way.

First, replace each set in the tuple with a set containing all states in the subtree of the corresponding rank-tree node. Then, sort these sets in ascending rank order and drop the ranks. For the macrostate $\left(\left\{q_{3}, q_{4}\right\}^{4},\left\{q_{2}\right\}^{2},\left\{q_{5}, q_{6}\right\}^{3},\left\{q_{1}\right\}^{1}\right)$ from Figure 5(b), the resulting sequence is $\left\{q_{1}, \ldots, q_{6}\right\},\left\{q_{2}, q_{3}, q_{4}\right\},\left\{q_{5}, q_{6}\right\},\left\{q_{3}, q_{4}\right\}$. The resulting sequence of sets $S_{1}, S_{2}, \ldots, S_{n}$ (which are different from the sets in the macrostate tuple) is essentially just a word over the alphabet $2^{Q}$ which satisfies the property that for all $i>1$, it holds that $\emptyset \neq S_{i} \subset S_{k}$ for some $k<i$ and all sets are pairwise different.

Notice that we can interpret such sequences of sets operationally, as a "recipe" describing how one can construct a rank-tree, when first starting just with the 
set of all states that are located in its nodes. The first set of the sequence is the set of all states that exist in the tree, and can be seen as a trivial rank-tree with all states in the root (which must have the rank 1). The following sets in the sequence can be read as a list of successive instructions, each set $S$ essentially saying: "find the current tree node that contains the states in $S$, create a new child node of that node (assigning it the new highest rank so far) and move the states in $S$ to the new node". Thereby, each additional set in the list "refines" the tree, until we get the exact rank-tree we are describing. Notice that each prefix of this sequence also describes a valid rank-tree with the same states, but with less nodes over which those states are distributed (see Figure 4).

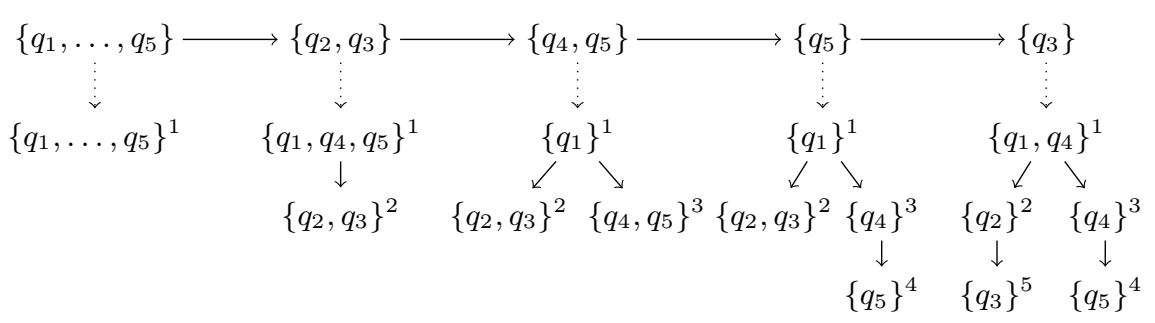

Fig. 4. Example illustrating the semantics of the set sequences which describe macrostates in terms of refining their rank-tree. The illustrated rank-trees at the bottom correspond to the different prefixes of the set sequence at the top.

Our approach now is to manage tries that store such sequences of sets, i.e., each trie node is labelled by a set $S \subseteq Q$ and a marker that indicates whether the sequence from the root down to the current node is stored in the trie (corresponds to a macrostate of the DPA that is constructed). The words that are added into this trie are exactly such sequences of sets as described above, so each trie node corresponds to the macrostate that is described by the set sequence from the root down to this node. In Figure 4, the set sequence at the top would be a single branch of the trie and each set would be a node of the trie that represents a specific macrostate, which is illustrated below the trie-node as the corresponding rank-tree.

To be more precise, for each set $U \subseteq Q$ of NBA states there is a separate trie (with the root labelled by $U$ ) that can be used to store all possible ranked slices (i.e. macrostates) with exactly the states from $U$, i.e., all macrostates $(\alpha, t)$ with $Q_{t}=U$. In practice, these tries are constructed on-the-fly to contain just the branches corresponding to macrostates that were already added to the DPA (see Figure 5 for an illustration).

Now we explain how to use these tries to aid the successor selection. Recall that in a transition of the determinization construction, during the merge operation adjacent sets can be grouped into intervals and merged together. The nondeterminism in the choice of intervals in this step allows for the multiple valid 


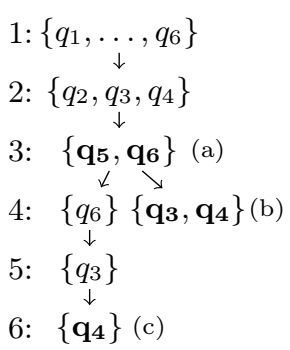

(a):
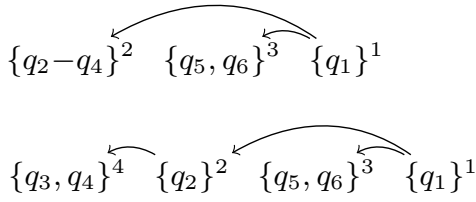

(c):

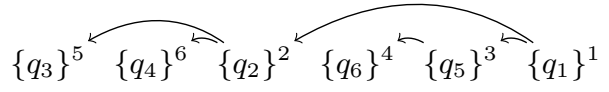

Fig. 5. The depicted trie on the left contains three macrostates that correspond to the marked trie nodes. The macrostate-tuples are depicted on the right, with ranks as superscripts and rank-tree edges (defined by parent index function $\uparrow$ ) connecting parents with children sets.

successors. In the view of macrostates as rank-trees, it is permitted to merge, for example, a whole subtree and/or multiple adjacent siblings into one node.

If $k$ is the dominating rank of the transition (obtained after performing prune), the constraints on merge ensure that certain nodes with more important (i.e., smaller) ranks, which are higher up in the rank-tree, more precisely - all nodes with a rank $<k$, cannot be changed at all. They keep exactly the same states and retain the same rank as before the merge. This is reflected in the set sequences described above by the fact that all valid successor macrostates agree on the prefix of length $k-1$, and only differ in the following sets that describe how the states are further distributed in the rank-tree. This means, that all valid successors must be located in the sub-trie that is rooted at the trie node reached after this common prefix of length $k-1$, which already significantly restricts the search space for a valid successor state.

Furthermore, each state $q$ that is not in a set of the rank-tree that has a rank $<k$ must eventually appear in some set at trie depth $\geq k$. If it does not, then in the macrostate described along the current trie branch, $q$ was never "promoted" downward in the rank-tree, violating the fact that nodes that have ranks $<k$ must not be modified by the merge operation, and especially must not contain additional states.

On the other hand, observe that one may skip the merge step completely (or do a trivial merge operation that collapses no sets), which corresponds to the successor agreeing with the Muller-Schupp construction. Because by performing non-trivial merges, the rank of each state $q$ (i.e., the rank of the set containing $q$ in the macrostate) may only decrease, the Muller-Schupp successor carries important information, namely for each state the highest rank of a node (of the rank-tree) that can have $q$ in its subtree, if the resulting macrostate shall be a valid successor. Translated to the trie view, this means that for each state of the NBA there is a deepest level of the trie where it may appear along the corresponding set sequence. 
We use the observations above in the following way. During a transition, we first compute the Muller-Schupp successor (i.e., skip merge) and transform the resulting macrostate into a sequence of sets, as described above.

First, we check whether the trie node reached by the $k-1$-prefix of the set sequence exists in the trie. If it does not, then there is no viable successor state existing in the automaton yet, as they all must share this prefix in the trie. Thus, we proceed by constructing the "default" successor, as prescribed by some merge strategy that is given as a parameter by the user (e.g. a merge that corresponds to the construction of Safra, Muller-Schupp, etc.), add this macrostate to the DPA and insert its set sequence into the trie, i.e., add the branch labelled by those sets and mark the final trie node reached by this set sequence, indicating that the corresponding macrostate is in the DPA.

If the trie node corresponding to the prefix does already exist in the trie, we search the trie using a depth-first search. This DFS follows existing branches in the subtrie, as long as they don't violate the constraints above (which can be computed from the Muller-Schupp successor we already constructed). Whenever a trie node is reached where these constraints are violated, the exploration of its sub-trie can be aborted, as the macrostates represented further below may not arise by a valid merge operation in the current transition.

Each trie node which does not violate the constraints, and is also marked, corresponds to a macrostate which already exists in the automaton and is a candidate for a valid successor. What remains to be ensured is that the candidate macrostate really can be obtained by merging adjacent non-empty sets. This is done using a simple unidirectional scan which compares the MullerSchupp successor (which we constructed in the beginning) to the current candidate macrostate (which already existed and just needs to be looked up). If a valid successor is identified during this exploration, then the DPA just gets a new transition to this already existing macrostate. Otherwise, we construct the "default" successor, add it to the DPA as the target of the current transition and also insert it into the trie, as described above.

From the practical point of view, all required steps can be carried out efficiently using bit-set operations, i.e. all set operations can be seen as constanttime operations. For all checks and computations only a linear (in the number of sets in a macrostate) number of set operations is required. Hence, the overall slowdown incurred by this optimized successor selection is very moderate. 


\section{F Additional examples}

The example in Figure 6 illustrates a case where heuristics presented in Section 4.2, which exploit the SCC-structure of the NBA, can be detrimental to state reduction and therefore using them requires greater care. One of the reasons is that by adding more determinization components in the modularized construction (Appendix A), it is possible that additional new macrostates are constructed due to the relocation of reached states of the NBA between the different tuples. Therefore, SCC-based heuristics should be only applied with sufficiently large SCCs, where a large reduction can be expected due to the separated handling.

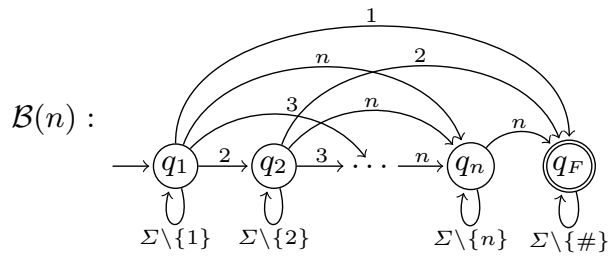

\begin{tabular}{l|l|ll}
$n$ & spot & def. & + sep \\
\hline 5 & 62 & 62 & 454 \\
6 & 126 & 126 & 2607 \\
7 & 254 & 254 & 17612
\end{tabular}

Fig. 6. $\mathcal{B}(n)$ is a family of automata with $n+1$ states for each $n$, where enabling some of the heuristics that enforce separated handling of SCCs of the NBA leads to more states. The table shows the resulting automata sizes from our prototype with just basic optimizations, and the same configuration with separation of all SCCs into different determinization components ( + sep) enabled. For any choice of the merge step, useless permutations of the states are introduced by the enforced separation, leading to the blowup.

The example in Figure 7 demonstrates how well the topological optimization complements the Muller-Schupp variant of the merge step in the determinization construction from Section 3.

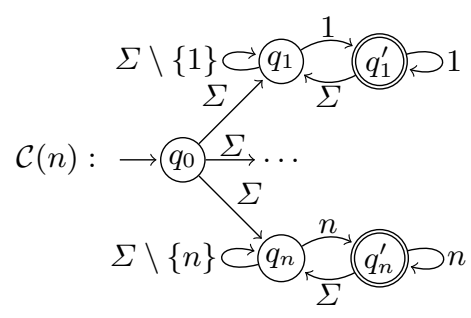

\begin{tabular}{l|l|lll}
$n$ & spot & def.+EI & $+\mathrm{T}$ & $+\mathrm{M}$ \\
\hline 2 & 3 & 6 & 4 & 1 \\
3 & 3 & 23 & 5 & 1 \\
4 & 3 & 126 & 6 & 1 \\
5 & 3 & 827 & 7 & 1 \\
6 & 3 & 6188 & 8 & 1
\end{tabular}

Fig. 7. $\mathcal{C}(n)$ is a family of universal automata with $2 n+1$ states for each $n$ on which using Muller-Schupp successors generates many useless states, which can be removed again using the topological optimization, as shown in the table. Together with our minimization we obtain the optimal automata. 